\title{
La educación sexual: ¿en tutorías o curricular?
}

\author{
Jose García Vázquez* \\ Consejería de Salud del Principado de Asturias
}

\author{
PALABRAS CLAVE \\ Educación sexual \\ Adolescentes \\ Escuelas
}

\section{KEYWORDS \\ Sex education \\ Adolescents \\ Schools}

\begin{abstract}
RESUMEN
La educación sexual es un derecho de la adolescencia que, sin embargo, sigue siendo voluntaria en las escuelas. Este estudio compara dos programas con un modelo de aplicación diferente (en tutorías vs. curricular), para así conocer qué tipo de intervención es más factible y realista. Se planteó un enfoque descriptivo longitudinal para comparar el proceso de "Ni ogros ni princesas" -NONP-, en tutorías, y "ESO por la Salud" -ExS-, curricular. Los indicadores se clasificaron en: valoración docente, participación, ejecución de actividades y opinión de personas coordinadoras. La información se recogió con cuestionarios para profesorado, fichas de seguimiento y entrevistas. La valoración docente fue similar en ambos programas, con porcentajes en torno al $90 \%$ de satisfacción y utilidad percibidas para el alumnado. NONP logró coberturas más altas, sobre todo en centros públicos (50 vs. $36 \%$ ). Las personas coordinadoras valoraron la continuidad de ambas iniciativas y destacaron la calidad del material didáctico y la implicación de alumnado y profesorado. Ambos programas fueron propuestas válidas, pero lastradas por la voluntariedad. A raíz de estos resultados y de un análisis más amplio de derechos, normas y evidencias, la educación sexual debería ser curricular y obligatoria.
\end{abstract}

\section{Sex education: tutoring or curricular?}

\section{ABSTRACT}

Sex education is a right of adolescence that, however, remains voluntary in schools. This study compares two programs with a different application model (tutoring vs. curricular), in order to know which type of intervention is more feasible and realistic. A longitudinal descriptive approach was proposed to compare the process of "Neither ogres nor princesses" -NONP-, tutoring, and "Compulsory Secondary Education for Health" -ExS-, curricular. The indicators were classified into: teacher evaluation, participation, carrying out activities and opinion of coordinators. The information was collected with questionnaires for teachers, follow-up sheets and interviews. The teacher evaluation was similar in both programs, with percentages around $90 \%$ of satisfaction and usefulness perceived for the students. NONP achieved higher coverage, especially in public schools (50 vs. $36 \%$ ). The coordinators valued the continuity of both initiatives and highlighted the quality of the teaching material and the involvement of students and teachers. Both programs were valid proposals, but burdened by volunteerism. Following these results and a broader analysis of rights, norms and evidence, sex education should be curricular and compulsory.

Consejería de Salud del Principado de Asturias

Autor de correspondencia: * Jose García Vázquez; josevazkez@yahoo.es

Recibido: 31/05/2021 - Aceptado: 08/07/2021

Revista de Formación del Profesorado e Investigación Educativa

Facultad de Formación del Profesorado y Educación

Universidad de Oviedo

Enero - Diciembre 2021

ISSN: 2340-4728

(c) (i) () $\Theta$ Esta obra está bajo una licencia internacional Creative Commons 


\section{Introducción}

La definición de la educación sexual ha ido cambiando con el paso de los años y, en la actualidad, se define como una actividad pedagógica, adaptada a cada edad y cultura, que utiliza información científica rigurosa, realista y sin prejuicios de valor, a nivel biológico, psíquico y social, para formar en sexualidad, entendiendo ésta como comunicación humana, fuente de salud, placer y afectividad (UNESCO, 2018).

La educación sexual de la infancia y adolescencia es un derecho, reconocido por la familia de Naciones Unidas (OMS, 2019; OPS y OMS, 2000; UNESCO, 2018; WHO, 2017) y priorizado en los Objetivos de Desarrollo Sostenible (United Nations, 2015), para construir una sociedad en la que mujeres y hombres puedan vivir en igualdad y sin discriminaciones (UNESCO, 2018). Asimismo, este derecho a una educación sexual de alta calidad ha sido ratificado por la comunidad internacional, en particular por la Comisión Internacional sobre Población y Desarrollo, que en su resolución 2012/117, insta a los gobiernos a proporcionar educación integral sobre sexualidad humana, salud sexual y reproductiva, e igualdad de género.

En esta línea, en nuestro país, la educación sexual cuenta con objetivos específicos en la Educación Secundaria Obligatoria (ESO), recogidos por la Ley Orgánica 8/2013, de 9 de diciembre, para la mejora de la calidad educativa, que es la que en la práctica está vigente en la actualidad. Entre otros, se reconoce la necesidad de "conocer y valorar la dimensión humana de la sexualidad en toda su diversidad". Sin embargo, a nivel curricular, la educación sexual no está incorporada como materia ni como contenido específico de una asignatura. Hay temas aislados en alguna materia, pero no se contempla en el currículo educativo ni en los libros de texto (García-Cabeza y Sánchez, 2013; Martínez et al., 2012).

Por último, la evidencia científica disponible determina que los programas escolares integrales de educación sexual, con enfoque de derechos y género, tienen efectos positivos significativos en conocimientos, actitudes y habilidades, y también en la tasas de Infecciones de Transmisión Sexual y embarazos no deseados (Rottach et al., 2009; UNFPA, 2014; UNESCO, 2012, 2015).

A pesar de estas normas y evidencias, los datos existentes indican que la educación sexual no está incorporada de forma efectiva ni universal, aunque hay propuestas para su implementación con programas de educación sexual no obligatorios, basados en la voluntariedad del profesorado y/o en talleres de personal externo (García et al., 2016; Lameiras et al., 2016; Larrañaga et al., 2014; Salvador, 2008; Venegas, 2011). No obstante, a pesar de la existencias de estos programas, en la mayoría de los casos, no se cuenta con información sobre la implementación y el alcance de los mismos, y la mayoría desaparece con el tiempo (García et al., 2016; Lameiras et al., 2016; Larrañaga et al., 2014; Ministerio de Sanidad, Política Social e Igualdad, 2011; Salvador, 2008; Venegas, 2011).

En Asturias, se han implementado diferentes iniciativas desde comienzo de los 90 y fruto de este trabajo y experiencia, en 2008, surgió "Ni ogros ni princesas" (NONP), un programa para la educación sexual en la ESO (Lena et al., 2009; García-Vázquez et al., 2009). Tras un año de pilotaje, se realizó una evaluación de proceso, que constató que era una intervención novedosa, con un gran nivel de ejecución, así como una alta participación, satisfacción y utilidad percibida por alumnado y profesorado (García-Vázquez et al., 2012). Más tarde, se llevó a cabo una evaluación de impacto, que detectó efectos positivos en los conocimientos, habilidades y conductas de las y los adolescentes (García-Vázquez et al., 2019). El programa, que era voluntario y se desarrollaba en tutorías, fue declarado como Buena Práctica por el Ministerio de Sanidad en 2018 y como Best Practice en Promoción de la Salud por la Comisión Europea en 2020.
Paralelamente, con el fin de mejorar y extender la "Educación y Promoción de la Salud en la escuela", una línea prioritaria y conjunta de las Consejerías de Salud y Educación de Asturias, se puso en marcha otro programa llamado "ESO por la salud" (ExS), en 2012 (Aguiar et al., 2014). Los contenidos de este programa eran más amplios en relación a la salud: bienestar emocional, alimentación, ejercicio, prevención del consumo de drogas y también educación sexual. De igual manera, se pilotó y tras unos resultados positivos, se implantó en Asturias a partir del curso 2013-14. Esta intervención, a diferencia de NONP, era curricular, es decir, que las actividades estaban diseñadas para abordar temas del currículo de diferentes materias, pero incluyendo contenidos de salud y educación sexual. Como ejemplos, en un tema curricular de Inglés como es la familia, se reflexionaba sobre la diversidad familiar, o en el estudio de "La Celestina" en Lengua y Literatura, se revisaban los mitos del amor romántico.

De este modo, en Asturias, convivieron en el tiempo dos programas con contenidos similares de educación sexual, NONP y ExS, pero con un modelo de aplicación diferente: tutorial vs. curricular.

Con el fin de avanzar en la incorporación real de la educación sexual en la escuela, este trabajo pretende comparar estos dos programas en términos de participación de alumnado, profesorado, cobertura en centros, valoración docente y ejecución de sus actividades. Se busca así conocer qué tipo de intervención es más factible y realista para contar con la mayor cantidad de información disponible para implementar con éxito la educación sexual en las escuelas.

\section{Metodología}

\section{Tipo de estudio}

Se planteó un estudio descriptivo longitudinal, combinando un enfoque cuantitativo y cualitativo, para comparar los resultados de proceso de dos programas de educación sexual con contenidos similares pero diferente forma de aplicación: tutorial (en tutorías) vs. curricular (en materias). Este enfoque encaja en el campo académico de estudio de la educación comparada, que examina la educación, en este caso, sexual, mediante la comparación de diferentes indicadores para contribuir a solucionar los problemas y mejorar la educación sexual de la población adolescente asturiana (Egido y Martínez-Usarralde, 2019).

\section{Las intervenciones}

Ambos programas incorporaban la educación sexual en la ESO con un enfoque de derechos y género: NONP proponía 48 sesiones de trabajo en el aula y ExS tenía 35, con los contenidos marcados en las directrices internaciones (WHO Regional Office for Europe y BZgA, 2010) y con el protagonismo del alumnado y profesorado en el proceso de aprendizaje. NONP se pilotó en el curso 2008-09, implantándose en Asturias en el 2009-10. A raíz de esta experiencia (García-Vázquez et al., 2012), se utilizó el mismo proceso para el diseño de ExS, que se pilotó en el curso 2012-13 y se implantó a partir del 2013-14. De este modo, ambos programas compartían características similares: tenían un material didáctico específico elaborado por profesorado de Asturias y un proceso con una serie de acciones para llevar a cabo en los centros educativos. Estas acciones eran: información a la comunidad educativa (cartas, folletos, reuniones...), formación del profesorado, sesiones del profesorado en el aula, talleres externos para el alumnado (que complementaban la labor del profesorado, no la sustituían) y sesión formativa para familias. Todas estas acciones eran voluntarias, salvo las sesiones del profesorado en el aula, que eran obligatorias y justificaban la participación e implementación del programa. El procedimiento para que los centros participasen en cada programa también era el mismo: oferta desde la Consejería 
de Educación al principio de curso e inscripción por parte de los centros con la aprobación del equipo directivo, del Consejo Escolar y con el nombramiento de una persona como coordinadora del programa en el centro.

Las diferencias entre ambas intervenciones eran fundamentalmente tres. En primer lugar, que NONP estaba diseñado para ser implementado en horas de tutoría mientras que en ExS, como ya se comentó, las actividades estaban diseñadas para ser llevadas a cabo dentro del currículo de siete materias: Ciencias de la Naturaleza, Educación Física, Lengua Castellana y Literatura, Ciencias Sociales, Inglés, Educación Ética y para la Ciudadanía, y Matemáticas. Otra diferencia entre ambos programas era que NONP era un programa integral de educación sexual mientras que en ExS, la educación sexual era una parte, pero había también otros temas de Educación para la Salud, ya mencionados previamente. Por último, la oferta de talleres en NONP era más amplia, con un taller de educación sexual con contenidos específicos por nivel de la ESO, mientras que en ExS solo se ofertaba un único taller genérico que el profesorado decidía en qué nivel se impartía.

\section{Indicadores, fuentes de información y población de estudio}

Los indicadores de este estudio se clasificaron en 4 categorías: valoración docente del programa, datos de participación, ejecución de las actividades y opinión de las personas coordinadoras.

1) Para la valoración docente, se pasó un cuestionario anónimo y autoadministrado al profesorado que participó en el programa en el pilotaje del mismo. Este pilotaje se llevó a cabo en el curso previo a la implantación del programa: en 12 centros en el curso 2008-09 en NONP y en 15 centros en el curso 2012-13 para ExS. Se seleccionó al 100\% del profesorado participante y se le invitó a cumplimentar el cuestionario: 185 docentes en NONP y 168 en ExS. La tasa de respuesta fue de $73 \%$ y del $68 \%$, respectivamente. Se utilizaron las siguientes variables en el cuestionario: satisfacción con la participación en el programa, dificultad de las actividades didácticas, satisfacción percibida en el alumnado, utilidad para el alumnado, interés de talleres externos y valoración del material didáctico. En todos los ítems, se utilizó una escala Likert.

2) Los datos de participación se recogieron mediante las fichas de seguimiento enviadas por los centros educativos a la Consejería de Educación como evaluación anual del curso académico. De este modo, para cada programa y curso escolar, se obtuvo el número de alumnado, profesorado (desglosado por sexo), familias y centros participantes. Los años de estudio fueron los cuatro primeros años de implantación de cada programa (tras el año de pilotaje): en NONP, desde el curso 2009-10 hasta el 2012-13; en ExS, del curso 2013-14 al 2016-17. La selección de cuatro cursos fue motivada porque esa es la duración de la Educación Secundaria Obligatoria.

3) En el caso de la ejecución de actividades, se utilizaron los datos de la ficha de seguimiento que cada centro enviaba a la coordinación de cada programa para su evaluación y se recogió el número de centros que llevó a cabo actividades de información a la comunidad educativa, sesiones del profesorado en el aula y sesiones para familias. Asimismo, se recogió el número medio de sesiones impartidas por el profesorado en el aula.

4) En relación con la opinión de las personas coordinadoras, se realizó una entrevista semiestructurada con preguntas abiertas para conocer los logros, dificultades y propuestas de mejora. En total fueron 131 entrevistas durante cuatro cursos escolares en NONP y 129 en ExS.

\section{Análisis de datos}

Se calcularon porcentajes de respuesta del profesorado para cada uno de los indicadores contemplados en la valoración do- cente. Para los datos de participación, se halló cobertura en Asturias de cada programa en relación con el alumnado y centros, utilizando como denominadores los datos correspondientes a cada curso escolar recopilados por el Ministerio de Educación y Formación Profesional (2021). Respecto a la ejecución de actividades, se calcularon los porcentajes sobre el total de centros participantes en cada programa. Por último, en las entrevistas a las personas coordinadoras, se hizo un análisis del discurso, se identificaron y clasificaron los temas, y se agruparon las respuestas en las categorías abiertas y excluyentes establecidas: logros del programa, dificultades y propuesta de mejora.

\section{Resultados}

\section{Valoración docente}

La valoración por parte del profesorado implicado fue similar en los dos programas (Ver Tabla 1): en torno al $90 \%$ señaló que las actividades didácticas eran fácilmente realizables, satisfactorias y útiles para el alumnado, y que el material didáctico era bueno. El interés de los talleres fue más alto en NONP ( $84 \%$ vs. $71 \%$ en ExS). La principal diferencia entre ambos programas fue en la satisfacción propia del profesorado con su participación en el programa: el 79\% del profesorado de NONP estuvo bastante/muy satisfecho frente al $96 \%$ de ExS.

Tabla 1. Porcentaje de respuesta del profesorado sobre satisfacción y utilidad percibida de los programas

\begin{tabular}{|l|c|c|}
\cline { 2 - 3 } \multicolumn{1}{l|}{} & $\begin{array}{c}\text { NONP } \\
(\%)\end{array}$ & $\begin{array}{c}\text { ExS } \\
(\%)\end{array}$ \\
\hline $\begin{array}{l}\text { Bastante/mucha satisfacción con su participa- } \\
\text { ción en el programa }\end{array}$ & 79 & 96 \\
\hline Actividades en aula fácilmente realizables & 93 & 96 \\
\hline $\begin{array}{l}\text { Bastante/mucha satisfacción percibida en el } \\
\text { alumnado }\end{array}$ & 90 & 90 \\
\hline Bastante/mucha utilidad para el alumnado & 90 & 91 \\
\hline $\begin{array}{l}\text { Los talleres externos le parecieron bastante/ } \\
\text { muy interesante }\end{array}$ & 84 & 71 \\
\hline El material didáctico es bastante/muy bueno & 90 & 86 \\
\hline
\end{tabular}

\section{Participación}

NONP (Ver Gráfico 1): El porcentaje de estudiantes que participó pasó del 14\% del alumnado de la ESO de Asturias en su primer año de implementación al $25 \%$ en el cuarto año (38\% del alumnado en centros públicos). Este aumento ocurrió también en el número de los centros educativos implicados: 21 a 41 (36\% de los centros de Secundaria). En el caso de la educación pública, el porcentaje de centros que participó en el último curso fue del $50 \%$. Asimismo, la participación del profesorado también aumentó desde 254 a 434 docentes (69\% eran mujeres). En el caso de las familias, que asistieron a la sesión formativa, se mantuvieron las cifras de forma más o menos estable en los cuatro años siguientes (en torno a las 200 personas/año).

ExS (Ver Gráfico 2): El porcentaje de estudiantes creció del 10\% al $21 \%$ en el cuarto año de implementación ( $27 \%$ del alumnado en centros públicos). Este aumento también ocurrió en el número de centros: de 27 a 37 (33\% de los centros de Secundaria). En el caso de la educación pública, el porcentaje de centros que participó en el último curso fue del $37 \%$. Asimismo, la participación del profesorado aumentó desde 168 a 298 docentes (70\% eran mujeres). En el caso de las familias, se produjo una evolución negativa, desde 207 personas formadas en el primer año a 95 en el último. 


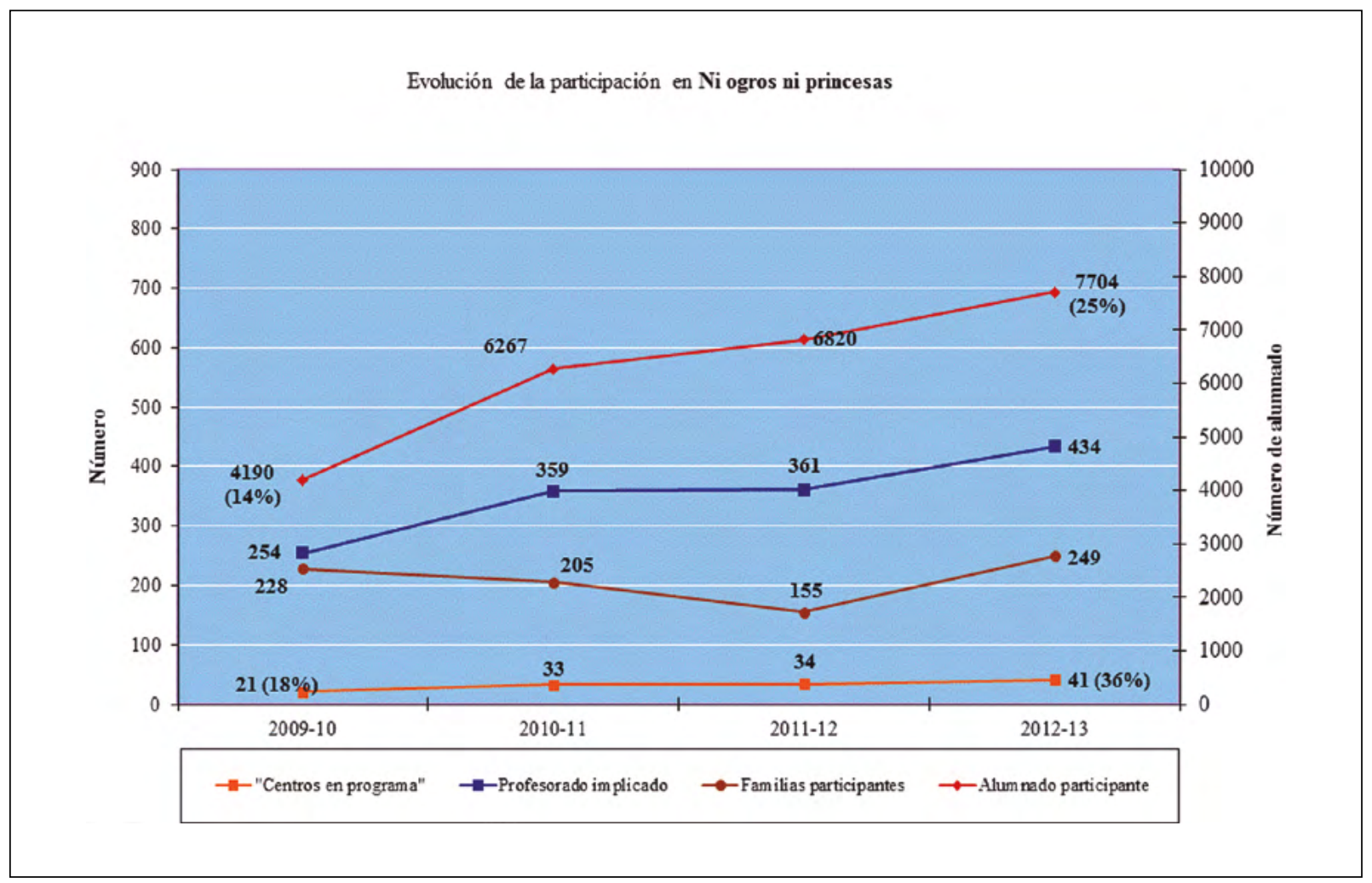

Gráfico 1. Número de personas y centros que participaron en el programa NONP, 2009-2013

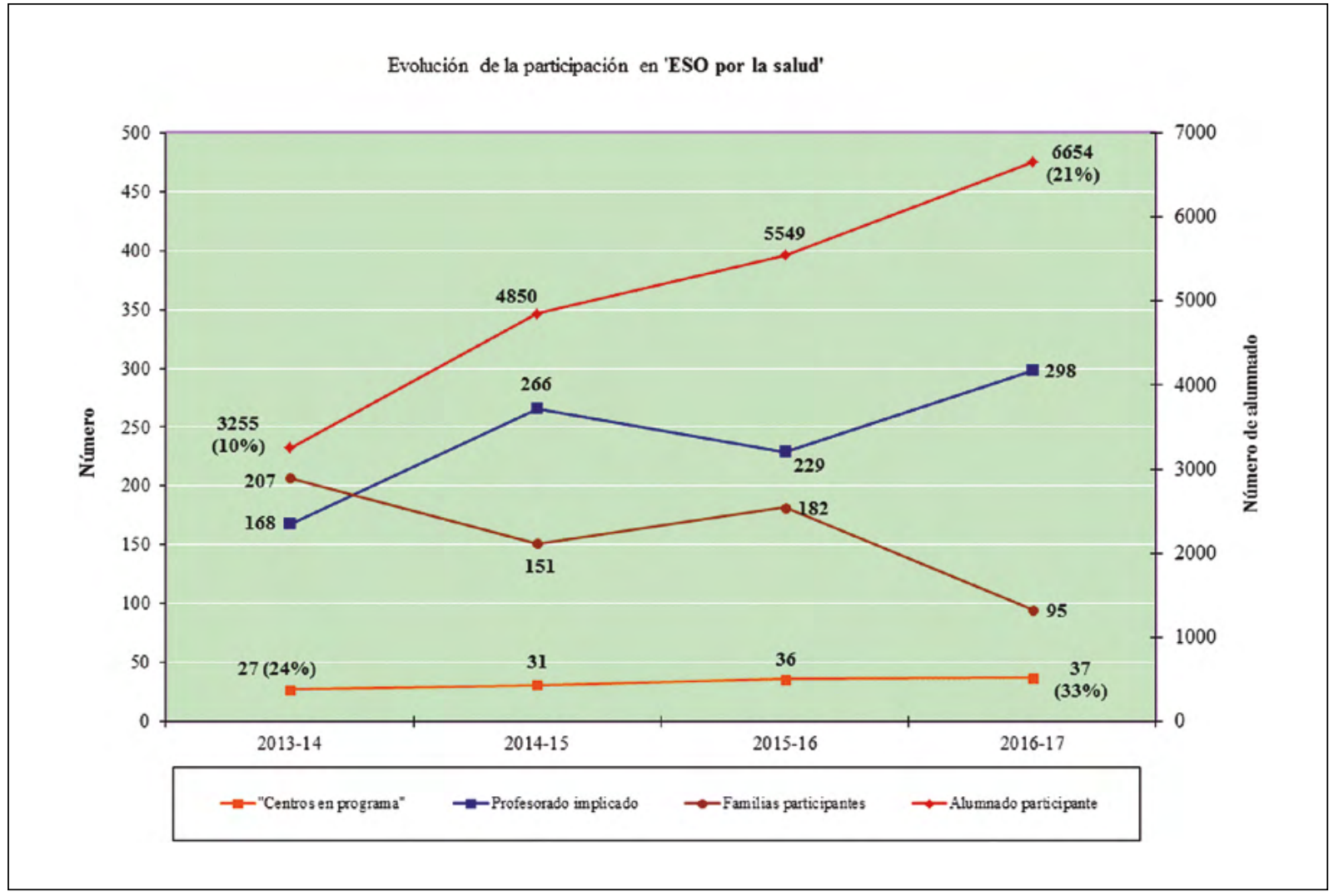

Gráfico 2. Número de personas y centros que participaron en el programa ExS, 2013-2017

\section{Ejecución de las actividades}

NONP: Las actividades de información a la comunidad educativa fueron realizadas de forma anual por la mayoría de los cen- tros, oscilando entre $91 \%$ de los centros que las realizó en el tercer año de implantación y el 100\% en el primero. En el 100\% de los centros, el profesorado llevó a cabo sesiones con el alumnado y el número medio anual de sesiones se mantuvo estable en 7 . En 
relación con las familias, el 76\% de los centros llevó a cabo la sesión formativa en el primer año y se redujo hasta un tercio de los centros en el cuarto año de implantación.

ExS: La cobertura anual de las actividades de información a la comunidad educativa fue mayoritaria: entre el $65 \%$ de los centros en el tercer año de implantación y el $93 \%$ en el primero. El profesorado realizó sesiones con su alumnado en el 100\% de los centros, pero el porcentaje de centros que desarrolló sesiones específicas de educación sexual osciló entre el $65 \%$ (primer año) y el 85\% (cuarto año). El número anual medio de sesiones de educación sexual fue entre 2 (segundo año) y 4 (último año de implantación). En torno al 50\% de los centros, llevó a cabo sesiones formativas con las familias, salvo en los últimos cursos que se realizaron solo en un tercio de los centros.

\section{Opinión de las personas coordinadoras}

Las personas coordinadoras valoraron en ambos programas como principales logros la implicación y satisfacción del alum- nado y del profesorado, así como la continuidad y consolidación de estos programas (Ver Tabla 2). En NONP, además señalaron cambios en conocimientos y actitudes de alumnado y profesorado, mientras que en ExS, se apuntó, como logro, la colaboración de profesorado de diferentes Departamentos.

En relación con las dificultades, se priorizaron la falta de implicación del profesorado y de las familias en ambos programas. La saturación de las horas de tutoría, donde se abordan muchos temas y se considera el "cajón de sastre", en NONP, y la resistencia de parte del profesorado para cambiar las actividades que ya tenía elaboradas de otros cursos e incorporar metodologías activas y participativas, en ExS, fueron otras de las dificultades planteadas.

Por último, entre las propuestas de mejora, se plantearon la continuidad de los programas, y más formación e implicación del profesorado. En NONP, también se insistió en la formación de equipos directivos y familias, mientras que en ExS en la colaboración de todos los Departamentos.

Tabla 2. Logros, dificultades y propuestas de mejora de las personas coordinadoras

\begin{tabular}{|c|c|c|}
\hline \multicolumn{3}{|c|}{ Logros } \\
\hline Ambos Programas & NONP & ExP \\
\hline $\begin{array}{l}\text {-Implicación y satisfacción de alumna- } \\
\text { do y profesorado. } \\
\text {-Organización del programa, a nivel de } \\
\text { contenidos y metodología. } \\
\text {-Calidad del material didáctico. } \\
\text {-Muy buena valoración de los talleres } \\
\text { externos. } \\
\text {-Continuidad y consolidación del pro- } \\
\text { grama. }\end{array}$ & $\begin{array}{l}\text { - “Normalización” de la educación se- } \\
\text { xual en el centro. } \\
\text {-Cambios en alumnado: aumento cono- } \\
\text { cimientos, mejora autoestima, mejora convi- } \\
\text { vencia, mejora respeto. } \\
\text {-Cambios en profesorado: mayor sen- } \\
\text { sibilización } \\
\text {-Introducción del programa en Progra- } \\
\text { mación General Anual y Plan de Acción Tu- } \\
\text { torial. }\end{array}$ & $\begin{array}{l}\text {-Propuestas curriculares adecuadas, úti- } \\
\text { les y fácilmente aplicables. } \\
\text {-Inclusión de la educación sexual en } \\
\text { otras materias y colaboración de diferentes } \\
\text { Departamentos. }\end{array}$ \\
\hline \multicolumn{3}{|c|}{ Dificultades } \\
\hline Ambos Programas & NONP & ExP \\
\hline $\begin{array}{l}\text {-Poca participación de parte del profe- } \\
\text { sorado: falta de tiempo, ausencia de forma- } \\
\text { ción, itinerancia. } \\
\text {-Escasa participación de familias. } \\
\text {-Excesiva oferta de programas y limita- } \\
\text { ciones del contrato programa. } \\
\text {-Falta de apoyo del equipo directivo. } \\
\text {-Homofobia y machismo entre parte del } \\
\text { alumnado y del profeso }\end{array}$ & $\begin{array}{l}\text {-Saturación de las tutorías con temas } \\
\text { diversos. }\end{array}$ & $\begin{array}{l}\text {-Resistencia de parte del profesorado a } \\
\text { cambiar sus actividades curriculares. } \\
\text {-Resistencia de parte del profesorado a } \\
\text { metodologías actividad y participativas en } \\
\text { el aula. }\end{array}$ \\
\hline \multicolumn{3}{|c|}{ Propuestas de mejora } \\
\hline Ambos Programas & NONP & ExP \\
\hline $\begin{array}{l}\text {-Continuidad. } \\
\text {-Propuestas didácticas novedosas y con } \\
\text { materiales audiovisuales. } \\
\text {-Formación vinculada al programa al } \\
\text { inicio del curso escolar. } \\
\text {-Reconocimiento para el profesorado } \\
\text { implicado. }\end{array}$ & $\begin{array}{l}\text {-Formación para el profesorado en el } \\
\text { propio centro educativo. } \\
\text {-Formación en igualdad y sexualidad } \\
\text { del equipo directivo. } \\
\text {-Propuestas alternativas para la forma- } \\
\text { ción de las familias. }\end{array}$ & $\begin{array}{l}\text {-Implicación de al menos un miembro } \\
\text { de cada Departamento y abrirlo a más ma- } \\
\text { terias. }\end{array}$ \\
\hline
\end{tabular}




\section{Discusión}

Este estudio pretendía comparar dos programas, con un modelo de aplicación diferente, para incorporar la educación sexual en la ESO. Los resultados muestran que la valoración docente de ambas experiencias fue muy positiva en todos los indicadores analizados, con porcentajes muy altos de satisfacción con el programa, tanto del profesorado como del alumnado, con el material didáctico y las actividades propuestas, y con la utilidad percibida de ambas intervenciones. La principal diferencia entre ambos programas fue la satisfacción propia del profesorado con su participación, que fue más alta en ExS, lo que posiblemente tenga que ver con que este era un programa más amplio, con otros temas de salud, mientras que NONP se centraba únicamente en educación sexual, que resulta un tema más complicado para el profesorado, ya que se puede sentir menos seguro (García-Vázquez et al., 2012).

Sin embargo, NONP llegó a más centros y alumnado. A pesar de que la evolución de ambos programas fue similar en los cuatro años estudiados, doblando prácticamente el número inicial de alumnado y profesorado implicado, el alcance en número y cobertura de centros fue mayor en NONP, que también se dobló, mientras que en ExS este incremento en centros fue aproximadamente del 40\%. Cabe destacar que NONP llegó al 50\% de los centros públicos de Secundaria de Asturias, con diferencias de más de 10 puntos porcentuales respecto a ExS. Este hecho quizá pueda explicarse por una oferta más amplia de talleres en NONP, un recurso de gran atractivo para alumnado y profesorado, y en una mayor resistencia de los centros concertados a iniciativas de educación sexual que, sin embargo son más receptivos a intervenciones más amplias donde la educación sexual es solo una parte y se puede no ejecutar, y de ahí el mayor número de centros concertados en ExS, en detrimento de los públicos (Lameiras et al., 2016; Salvador, 2008; Venegas, 2011).

Este hecho se refleja precisamente en el desarrollo de las acciones de los programas. Mientras que las que eran voluntarias, se llevaron a cabo de forma mayoritaria, salvo la formación de familias que dejó de impartirse, la única acción obligatoria, sesiones del profesorado, no se realizó en todos los centros de ExS cuando se analiza el tema impartido: entre el 65 y el $85 \%$ de los centros, en función del año, refirió haber impartido sesiones de educación sexual. Este dato se refleja también en el número medio de sesiones impartidas por año: entre 2 y 4 en ExS vs. 7 en NONP. No obstante, hay que señalar que la incorporación de sesiones de educación sexual en ExS fue aumentando año a año.

La opinión de las personas coordinadoras ayuda a explicar también estos resultados: hay resistencias por parte del profesorado en ambos programas, falta de información y formación de profesorado, equipos directivos y familias, y actitudes en ocasiones todavía machistas y homófobas en los centros educativos, lo que sin duda dificulta la incorporación de la educación sexual. Estas dificultades se detectan también en otras investigaciones, junto con la voluntariedad, el protagonismo externo, la ausencia de apoyo institucional o la falta de apoyos de libros de texto, entre otras (García-Cabeza y Sánchez, 2013; García et al., 2016; Lameiras et al., 2016; Larrañaga et al., 2014; Martínez et al., 2012; Ministerio de Sanidad, Política Social e Igualdad, 2011).

En este contexto, la continuidad de estas iniciativas y la formación continua, recogidas como propuestas de mejora de las personas coordinadoras, son necesarias para mantener y mejorar la educación sexual mientras esta sea voluntaria, para así aumentar la sensibilización y formación de la comunidad educativa. Asimismo, también se perciben logros y cambios, más centrados en el proceso en el caso de ExS y con más resultados en el caso de NONP, lo que posiblemente tenga que ver con la concreción de los contenidos de este programa en un único tema: la educación sexual.
En resumen, la cobertura de ambos programas fue alta, teniendo en cuenta que eran intervenciones nuevas, que requieren de tiempo para asentarse y darse a conocer entre el profesorado y en los centros educativos, y no menos importante, que eran voluntarias. Asimismo, las acciones planteadas se llevaron a cabo en ambos programas, quizá porque estaban adaptadas al funcionamiento de los centros, tal vez porque eran fácilmente aplicables. En cualquier caso, el proceso propuesto se ejecutó y confirmó la aceptación de las dos intervenciones. Hubo además una valoración docente positiva y una apuesta por la continuidad de quienes coordinaban.

A raíz de estos resultados, se puede concluir, por tanto, que ambos programas fueron propuestas innovadoras y válidas para los centros educativos, pero NONP contribuyó más a incorporar la educación sexual en las escuelas, al ser una intervención centrada exclusivamente en este tema. Esta premisa, y no tanto el modo de aplicación parece ser más relevante a la hora de introducir la educación sexual en las escuelas. De otra parte, el seguimiento posterior de NONP (ya que es un programa que continúa vigente), ha permitido conocer cómo evolucionó la cobertura en años siguientes, que siguió aumentado hasta alcanzar un pico máximo ( $45 \%$ del alumnado y $60 \%$ de los centros públicos de Secundaria), para luego estabilizarse en esos datos (Consejería de Salud de Asturias, 2021).

Estas cifras, que pueden considerarse elevadas y positivas, ocultan sin embargo varias consideraciones a tener en cuenta: por un lado, la calidad de la intervención no se evaluó, tal y como se recoge más adelante en limitaciones, por lo que no se sabe si la educación sexual recibida por el alumnado es adecuada; por otro, el número medio de sesiones anuales recibidas por este alumnado es limitado (7), lo que sin duda son pocas horas si se quiere garantizar una educación sexual integral. Ambas cuestiones se suman a una tercera que quizá sea la más contundente: a pesar del gran esfuerzo del profesorado, centros educativos e instituciones promotoras, la mayoría del alumnado de Secundaria de Asturias no recibe una educación sexual integral, a pesar de ser un derecho, una norma y una intervención beneficiosa para la salud.

Por tanto, para dar respuesta a la pregunta planteada en el título, hay varias consideraciones a tener en cuenta. En primer lugar, ambos programas se ejecutaron, con satisfacción del alumnado y profesorado, e hicieron posible la educación sexual en la escuela, aunque de forma limitada. En este sentido, es posible la educación sexual impartida por el profesorado. Por otro lado, atendiendo a las coberturas, la educación sexual curricular o en tutoría no fue universal y un amplio porcentaje del alumnado no la recibió, a pesar de ser un derecho, una norma y una evidencia científica. Por último, el alcance de la educación sexual en el alumnado que la recibió fue muy limitado, con pocas horas recibidas sobre este tema. En base a los datos analizados y a estas consideraciones, se puede concluir que la respuesta a la pregunta planteada en el título no aparece recogida de forma completa en el mismo, ya que es la siguiente: educación sexual (curricular o en tutorías) obligatoria. En este sentido, el tiempo de la voluntariedad ya pasó, y estos programas, y otros, pueden ser un buen punto de partida para avanzar hacia una educación sexual universal y para diseñar una educación sexual obligatoria, tal y como se recomienda a nivel internacional (WHO Regional Office for Europe y BZgA, 2010; UNESCO, 2018). Por tanto, es el momento de que la educación sexual, con un enfoque de derechos y género, sea una materia o parte de una, con contenidos amplios y específicos (EWEC, 2015; OMS, 2016; UNESCO, 2012, 2017, 2018; WHO Regional Office for Europe y BZgA, 2010), tal y como se hace en otros países con resultados positivos (UNESCO, 2012, 2017, 2018; WHO Regional Office for Europe y BZgA, 2010). Como conclusión, la respuesta final, a raíz de los datos de este estudio y haciendo un análisis más amplio, es educación sexual curricular obligatoria. En este punto, se ha aprobado recientemente una nueva legislación educativa, la ley Orgánica 3/2020, de 29 de diciem- 
bre, por la que se modifica la Ley Orgánica 2/2006, de 3 de mayo, de Educación. Su aplicación efectiva, a partir del curso 2021-22, puede ser una oportunidad para avanzar en la incorporación curricular y obligatoria de la educación sexual.

La educación sexual es imprescindible para promover la salud sexual y el bienestar de las personas, para que puedan decidir con autonomía sobre su vida sexual y reproductiva, y disfrutar de una vida sexual, placentera, segura y saludable, libre de estereotipos de género y de cualquier tipo de violencia o discriminación (Duberstein y Maddow-Zimet, 2011; UNESCO, 2018; WHO Regional Office for Europe y BZgA, 2010). La responsabilidad de que niños, niñas y adolescentes ejerzan su derecho a la educación sexual corresponde a las autoridades, en especial a las educativas y sanitarias, y por supuesto también a las familias (WHO Regional Office for Europe y BZgA, 2010). Estas instituciones deben dar información, formación y valores para que las y los jóvenes puedan tomar el control sobre su salud para así poder mejorarla (Breuner et al., 2016; Oringanje et al., 2016; WHO Regional Office for Europe, y BZgA, 2010).

Es una cuestión de derechos y salud pública (Starrs et al., 2018), tal y como recogen los Objetivos de Desarrollo Sostenible (UN, 2015), y por tanto es una necesidad urgente para mejorar el bienestar, la salud y la educación de nuestras niñas, niños y adolescentes.

\section{Limitaciones}

Este estudio contó con varias limitaciones. En primer lugar, las propias del uso de cuestionarios y entrevistas, por los posibles sesgos en las respuestas de personas implicadas en la educación sexual, ya que no se recogieron las opiniones del profesorado que no participó en los programas. Por otro lado, como ya se adelantó, no se recogió la calidad de las intervenciones, ni la fidelidad a las sesiones planteadas ni la metodología implementada en el aula, ya que no eran objetivos de este estudio, que solo se centraba en la aplicabilidad y aceptación de la educación sexual. No obstante, estas cuestiones podrían afectar a los parámetros estudiados. En esta línea, tampoco se recogió la opinión directa del alumnado, elemento clave de todo el proceso, ni de las familias, otro actor imprescindible de la educación sexual de la infancia y adolescencia.

\section{Agradecimientos}

El presente trabajo ha sido financiado por el Programa de Educación Afectivo-Sexual para Asturias, de la Consejería de Salud del Principado de Asturias.

\section{Referencias}

Aguiar, S., Alonso, J.J., Blanco, A.G., Ceballos, A., Fernández, M.J., Frechilla, A., García, E., García-Vázquez, J., Lena, A., Pérez, M.E., Rodríguez, R., Suárez, C. y Vallina, M. (2014). ESO por la Salud. Material curricular para la coeducación y la salud en Secundaria. Consejería de Educación, Cultura y Deporte del Principado de Asturias. https://www.astursalud.es/noticias/-/noticias/eso-por-la-salud

Breuner, C.C. y Mattson, G. (2016). Sexuality Education for Children and Adolescents. Pediatrics, 138, e320161348. https://doi. org/10.1542/peds.2016-1348

Consejería de Salud del Principado de Asturias. (2021). Astursalud. Ni ogros ni princesas. https://www.astursalud.es/noticias/-/ noticias/ni-ogros-ni-princesas

Duberstein, L. y Maddow-Zimet, I. (2011). Consequences of sex education on teen and young adult sexual behaviors and outcomes. Journal of Adolescent Health, 51, 332-338. https://doi.org/10.1016/j.jadohealth.2011.12.028
Egido, I. y Martínez-Usarralde, M.J. (2019). La educación comparada, hoy. Editorial Síntesis.

EWEC. (2015). The global strategy for women's, children's and adolescents' health (2016-2030). United Nations. https://www. everywomaneverychild.org/global-strategy/

García-Cabeza, B. y Sánchez, A. (2013). Sex education representations in Spanish combined biology and geology textbooks. International Journal of Science Education, 35, 1725-1755. http:// dx.doi.org/10.1080/09500693.2013.795669

García-Vázquez, J., Lena, A., González, C. y Blanco A.G. (2009). Ni ogros ni princesas. Programa para la educación sexual en la ESO. Gobierno del Principado de Asturias. https://www.astursalud.es/documents/35439/39939/Ni+ogros+ni+princesas\%2C+programa+edicion+anterior.pdf/2c586049-cd57-2597-8e43$5 \mathrm{c} 3 \mathrm{e} 28 \mathrm{cf} 4589 \mathrm{t} \mathrm{t}=1621241558184$

García-Vázquez, J., Lena, A. y Suárez, O. (2012). Evaluación de proceso del programa de educación afectivo-sexual Ni ogros ni princesas. Global Health Promotion, 28, 109-115. http://dx.doi. org/10.1177/1757975912441224

García-Vázquez, J., Quintó, L. y Agulló-Tomás, E. (2019). Impact of a sex education programme in terms of knowledge, attitudes and sexual behaviour among adolescentes in Asturias (Spain). Global Health Promotion, 27, 122-130. https://doi. org/10.1177/1757975919873621.

García, Y., Aguilera, E.M., Reyero, M. y Martín S. (2016). Deficiencias e inequidad en los servicios de salud sexual y reproductiva en España. Alianza por la Solidaridad et al. https://www.medicosdelmundo.org/file/275/download?token=V9NAISnE

Lameiras, M., Carrera, M.V. y Rodríguez, Y. (2016). Caso abierto: la educación sexual en España, una asignatura pendiente. En V. Gavidia (Ed.), Los ocho ámbitos de la Educación para la Salud en la Escuela (pp. 197-210). Tirant Humanidades.

https://www.uv.es/comsal/pdf/librocomsal.pdf

Larrañaga, I., Martín, U. y Bacigalupe, A. (2014). Salud sexual y reproductiva, y crisis económica en España. Informe SESPAS 2014. Gaceta Sanitaria, 28, 109-115. https://gacetasanitaria.org/es-salud-sexual-reproductiva-crisis-economica-articulo-S0213911114000880

Lena, A., González, A., Fernández, A.B., Blanco, A.G., Fernández, A.I., Suárez, A.M., Silva, E., Rubio, M.D. y Mier, M. (2009). Ni ogros ni princesas. Guía para la educación sexual en la ESO. Gobierno del Principado de Asturias. https:/www.astursalud.es/ documents/35439/39936/Ni+ogros+ni+princesas\%2C+guia+para+el+profesorado.pdf/620fab11-27f4-e377-7dcf-8cdcc4f1a8b$\mathrm{d} ? \mathrm{t}=1612361020028$

Martínez, J.L., Carcedo, R.J. y Fuertes, A. (2012). Sex education in Spain: Teacher's views obstacles. Sex Education: Sexuality, society and learning, 12, 425-436. http://dx.doi.org/10.1080/1468 1811.2012.691876

Ministerio de Educación y Formación Profesional. (2021). Anuario estadístico. Las cifras de la educación en España. https://www. educacionyfp.gob.es/servicios-al-ciudadano/estadisticas/indicadores/cifras-educacion-espana.html

Ministerio de Sanidad, Política Social e Igualdad. (2011). Estrategia Nacional de Salud Sexual y Reproductiva. Gobierno de España.

https://www.mscbs.gob.es/organizacion/sns/planCalidadSNS/ pdf/equidad/ENSSR.pdf

OMS. (2016). Estrategia mundial del sector de la salud contra las infecciones de transmisión sexual 2016-2021: Hacia el fin de las ITS. Organización Mundial de la Salud. https://www.who.int/reproductivehealth/publications/rtis/ghss-stis/es/

OMS. (2019). Recomendaciones de la OMS sobre salud y derechos sexuales y reproductivos de los adolescentes. Organización Mundial de la Salud.

https://www.who.int/reproductivehealth/publications/adolescent-srhr-who-recommendations/es/

OPS y OMS. (2000). Promoción de la salud sexual: Recomendaciones para la acción. Organización Panamericana de la Salud y Or- 
ganización Mundial de la Salud. https://iris.paho.org/handle/10665.2/51672

Oringanje, C., Meremikwu, M.M., Eko, H., Esu, E., Meremikwu, A., y Ehiri, J. (2016). Interventions for preventing unintended pregnancies among adolescents. Cochrane Database of Systematic Reviews, 2, Art. №: CD005215. https://doi. org/10.1002/14651858.CD005215.pub3

Rottach, E., Schuler, S.R. y Hardee, K. (2009). Gender perspectives improve reproductive health outcomes: New evidence. US Agency for International Development. https://www.prb.org/wp-content/uploads/2021/01/030520210-genderperspectives.pdf

Salvador, T. (2008). Diagnóstico de situación sobre avances conseguidos, necesidades y retos en promoción y educación para la salud en la escuela en España. Ministerio de Educación y Ministerio de Sanidad y Consumo. https://sede.educacion.gob.es/ publiventa/informe-diagnostico-de-situacion-sobre-avances-conseguidos-necesidades-y-retos-en-promocion-y-educacion-para-la-salud-en-la-escuela-en-espana/educacion-infantil-y-primaria-salud-publica/15121

Starrs, A.M, Ezeh, A.C., Barker, G., Basu, A., Bertrand, J., Blum, R., Coll-Seck, A., Grover, A., Laski, L., Roa, M., Sathar, Z., Say, L., Serour, G., Singh, S., Stenberg, K., Temmerman, M., Biddlecom, A., Popinchalk, A., Summers, C. y Ashford, L. (2018). Accelerate progress-sexual and reproductive health and rights for all: report of the Guttmacher-Lancet Commission. Lancet, 391, 2642-92. https://www.thelancet.com/commissions/sexual-and-reproductive-health-and-rights

UNESCO. (2012). Programas escolares de educación sexual. Análisis de los costos y la relación costo-eficacia en seis países. Organización de las Naciones Unidas para la Educación, la Ciencia y la Cultura. https://www.icmujeres.gob.mx/wp-content/uploads/2020/05/ UNESCO-costo_eficacia_educacion_sexual.pdf
UNESCO. (2015). Emerging evidence, lessons and practice in comprehensive sexuality education: a global review. Organización de las Naciones Unidas para la Educación, la Ciencia y la Cultura. https://unesdoc.unesco.org/ark:/48223/pf0000243106

UNESCO. (2017). Estrategia de la UNESCO sobre la educación para la salud y el bienestar: contribución a los Objetivos de Desarrollo Sostenible. Organización de las Naciones Unidas para la Educación, la Ciencia y la Cultura.

https://unesdoc.unesco.org/ark:/48223/pf0000246453_spa

UNESCO. (2018). International technical guidance on sexuality education: an evidence-informed approach. Organización de las Naciones Unidas para la Educación, la Ciencia y la Cultura. https:// unesdoc.unesco.org/ark:/48223/pf0000260770_eng

UNFPA. (2014). Operational guidance for comprehensive sexuality education: A focus on human rights and gender. United Nations Population Fund. https://www.unfpa.org/publications/unfpa-operational-guidance-comprehensive-sexuality-education

UN. (2015). Transforming our world: the 2030 Agenda for Sustainable Development. United Nations. https://sdgs.un.org/2030agenda

Venegas, M. (2011). El modelo actual de educación afectivo sexual en España. El caso de Andalucía. Revista Iberoamericana de Educación, 55, 1-10. https://doi.org/10.35362/rie5531592

WHO Regional Office for Europe y BZgA. (2010). Standards for Sexuality Education in Europe: A Framework for Policy Makers, Educational and Health Authorities and Specialists. Federal Centre for Health Education, BZgA. https://www.bzga-whocc.de/en/ publications/standards-for-sexuality-education/

WHO. (2017). Leading the realization of human rights to health and through health: report of the high-level working group on the health and human rights of women, children and adolescents. World Health Organization. https://apps.who.int/iris/handle/10665/255540 